\title{
INVARIANCE OF THE DISTRIBUTIONAL CURVATURE OF THE CONE UNDER SMOOTH DIFFEOMORPHISMS
}

\author{
J.A.VICKERS AND J.P.WILSON
}

\begin{abstract}
An explicit calculation is carried out to show that the distributional curvature of a 2-cone, calculated by Clarke et al. (1996), using Colombeau's new generalised functions is invariant under non-linear $C^{\infty}$ coordinate transformations.
\end{abstract}

\section{INTRODUCTION}

Recently, Colombeau's theory of new generalised functions (Colombeau, 1984) has been applied to give a distributional interpretation to physical quantities in General Relativity that are calculated by non-linear processes, such as the distributional curvature of cosmic strings (Clarke et al, 1996), the Ultra-relativistic Riessner Nordstrøm field (Steinbauer, 1997) and the energy-momentum tensor of the Kerr solution (Balasin, 1997). One of the major problems of using the full Colombeau algebra, which undermines its usefulness in a covariant physical theory such as General Relativity is that it is not of a coordinate invariant construction; that is given a diffeomorphism $\mu: \Omega \rightarrow \Omega^{\prime}$ between two open sets, there is no natural way of defining a map $\tilde{\mu}^{*}: \mathcal{E}\left(\Omega^{\prime}\right) \rightarrow \mathcal{E}(\Omega)$ which preserves the concepts of moderate and null functions, and commutes with the canonical embedding (smoothing convolution) $\iota: \mathcal{D}^{\prime}(\Omega) \rightarrow \mathcal{E}(\Omega)$.

In this paper we shall return to the calculations of Clarke et al. (1996) and we shall explicitly show that the distributional curvature is independent of any non-linear $C^{\infty}$ coordinate transformation that we might carry out on the metric. This will provide evidence for a covariant formalism of Generalised functions

\section{The Distributional CURVATURE OF COSMiC STRINGS}

The distributional curvature of the cone with a deficit angle of $2 \pi(1-A)$, whose metric may be written as

$$
\begin{aligned}
& g_{a b}=\frac{1}{2}\left(1+A^{2}\right) \delta_{a b}+\frac{1}{2}\left(1-A^{2}\right) m_{a b} \\
& m_{a b}=\left(\begin{array}{cc}
\frac{x^{2}-y^{2}}{x^{2}+y^{2}} & \frac{2 x y}{x^{2}+y^{2}} \\
\frac{2 x y}{x^{2}+y^{2}} & -\frac{x^{2}-y^{2}}{x^{2}+y^{2}}
\end{array}\right)
\end{aligned}
$$

was recently calculated by Clarke et al. (1996) by using Colombeau's theory of generalised functions to overcome the problem of assigning distributional interpretations to products of distributions. It was shown to be

$$
R \sqrt{g}=4 \pi(1-A) \delta^{(2)}(x, y)
$$

This result implies that a thin cosmic string, whose exterior metric is

$$
d s^{2}=-d t^{2}+d r^{2}+A^{2} r^{2} d \phi^{2}+d z^{2}
$$

has a mass per unit length of $2 \pi(1-A) / A$.

The process used to calculate the curvature first involves an embedding of the metric $g_{a b}$ into $\mathcal{E}_{M}\left(\mathbb{R}^{2}\right)$;

$$
\begin{aligned}
& \iota: g_{a b} \mapsto \widetilde{g_{a b}} \\
& \widetilde{g_{a b}}(\Phi, x, y)=\int g_{a b}(x+\varepsilon \xi, y+\varepsilon \eta) \Phi(\xi, \eta) d \xi d \eta
\end{aligned}
$$

PACS: 0420, 1127

Keywords: Colombeau algebras, Conical singularities. 
The generalised function Ricci curvature $\tilde{R} \sqrt{\tilde{g}}$ is then is calculated from the metric $\widetilde{g_{a b}}$ in the usual manner, and finally it is shown that this curvature is equivalent to the distribution $4 \pi(1-A) \delta^{(2)}$ in the sense of weak equivalence; that is that for each $\Psi \in \mathcal{D}\left(\mathbb{R}^{2}\right)$

$$
\lim _{\varepsilon \rightarrow 0} \int \tilde{R} \sqrt{\tilde{g}} \Psi d x d y=\Psi(0,0)
$$

for $\Phi \in \mathcal{A}_{q}\left(\mathbb{R}^{2}\right)$, for large enough $q \in \mathbb{N}$.

The theory of General Relativity is however a covariant theory, so we would like these results to make sense in the context of coordinate invariance; that is given a $C^{\infty}$ diffeomorphism;

$$
\begin{aligned}
& \mu: \mathbb{R}^{2} \rightarrow \mathbb{R}^{2} \\
& \left(x^{\prime}, y^{\prime}\right)=\mu(x, y)
\end{aligned}
$$

the following diagram commutes;

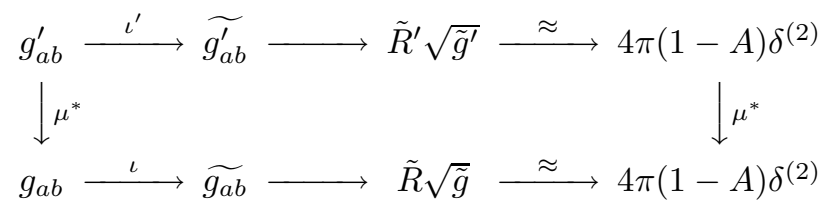

where $\mu^{*}$ denotes the appropriate tensor or density transformation laws

Since the full Colombeau algebra is not a coordinate invariant construction and the embedding $\iota$ does not commute with $\mu$, it will only make sense to talk about coordinate invariance at the level of distribution theory; this will mean that we must follow the approach of carrying out the coordinate transformation on the metric $g_{a b}^{\prime}$ to form a new metric $g_{a b}$, explicitly calculate the distributional Ricci scalar density for the new metric and show that it is transforms as a scalar density of weight +1 from the Ricci scalar density that corresponded to $g_{a b}^{\prime}$.

We first apply $C^{\infty}$ diffeomorphism $\mu: \mathbb{R}^{2} \rightarrow \mathbb{R}^{2}$ to the primed version of the metric (1); The fact that Colombeau's theory is manifestly invariant under linear coordinate transformations means that without loss of generality, it may be assumed that $\mu$ is of the form

$$
\begin{aligned}
& x^{\prime}=x+f(x, y) \\
& y^{\prime}=y+g(x, y)
\end{aligned}
$$

where $f$ and $g$ are $C^{\infty}$ and $O\left(x^{2}+y^{2}\right)$.

On applying (2) to (1) we are able to write

$$
g_{a b}(x, y)=\frac{1}{2}\left(1+A^{2}\right) l_{a b}(x, y)+\frac{1}{2}\left(1-A^{2}\right) m_{a b}(x, y)
$$

where

and

$$
\begin{aligned}
l_{a b} & =\frac{\partial x^{\prime c}}{\partial x^{a}} \frac{\partial x^{\prime d}}{\partial x^{b}} \delta_{c d} \\
m_{a b} & =\frac{\partial x^{\prime c}}{\partial x^{a}} \frac{\partial x^{\prime}}{\partial x^{b}} m_{c d}^{\prime}
\end{aligned}
$$

$$
\begin{aligned}
m_{a b}^{\prime} & =\left(\begin{array}{cc}
\frac{(x+f)^{2}-(y+g)^{2}}{(x+f)^{2}+(y+g)^{2}} & \frac{2(x+f)(y+g)}{(x+f)^{2}+(y+g)^{2}} \\
\frac{2(x+f)(y+g)}{(x+f)^{2}+(y+g)^{2}} & -\frac{(x+f)^{2}-(y+g)^{2}}{(x+f)^{2}+(y+g)^{2}}
\end{array}\right) \\
\frac{\partial x^{\prime a}}{\partial x^{b}} & =\left(\begin{array}{cc}
1+f_{, x} & f_{, y} \\
g_{, y} & 1+g_{, y}
\end{array}\right)
\end{aligned}
$$

Next we shall smooth $g_{a b}$ with a kernel $\Phi \in \mathcal{A}_{0}\left(\mathbb{R}^{2}\right)$ having a support in a radius of

$$
R_{0}=\left\{\left(x^{2}+y^{2}\right)^{1 / 2}|| \Phi(x, y) \mid>0\right\} .
$$


The functions $l_{a b}$, consisting of products of the smooth Jacobian components, will be $C^{\infty}$ and so may be identified with their smoothings in $\mathcal{E}_{M}\left(\mathbb{R}^{2}\right)$.

The matrix $m_{a b}$, containing the singular contributions to the metric, may be expressed as

$$
m_{a b}=\left(1+2 \frac{x f+y g}{x^{2}+y^{2}}+\frac{f^{2}+g^{2}}{x^{2}+y^{2}}\right)^{-1}\left(\begin{array}{cc}
\frac{x^{2}-y^{2}+f_{11}}{x^{2}+y^{2}} & \frac{2 x y+f_{12}}{x^{2}+y^{2}} \\
\frac{2 x y+f_{21}}{x^{2}+y^{2}} & \frac{y^{2}-x^{2}+f_{22}}{x^{2}+y^{2}}
\end{array}\right)
$$

where the $f_{a b}(x, y)$ are $C^{\infty}$ and $O\left(r^{3}\right)$ functions. It may be observed that the components of the above matrix may be expressed as linear combinations of real and imaginary parts of complex-valued functions having the form

$$
m(x, y)=\frac{e^{2 i \phi}+m_{1}(x, y) e^{-2 i \phi}+m_{2}(x, y)+m_{3}(x, y) e^{2 i \phi}}{1+m_{4}(x, y) e^{-2 i \phi}+m_{5}(x, y)+m_{6}(x, y) e^{2 i \phi}}
$$

where we define the polar coordinates $(r, \phi)$ by

$$
x=r \cos \phi, \quad y=r \sin \phi
$$

and the $m_{k}(x, y)$ are $C^{\infty}$ and $O(r)$. Therefore we may calculate $\widetilde{m_{a b}}$ by smoothing $h(x, y)$.

The expression for $m(x, y)$ may be expanded as

$$
m(x, y)=e^{2 i \phi}+r \sum_{k= \pm 1, \pm 3} \alpha_{k} e^{k i \phi}+r^{2} p(x, y)
$$

where the $\alpha_{k}$ are constants and $p(x, y)$ is $O\left(r^{0}\right)$, continuous everywhere and $C^{\infty}$ everywhere except at $r=0$. We may smooth this expansion termwise and obtain estimates for the smoothings. Certainly the $r e^{ \pm i \phi}$ are $C^{\infty}$ and may be identified with their smoothings. For other terms, the results in the appendix will give

$$
\begin{gathered}
\widetilde{e^{2 i \phi}}= \begin{cases}C_{1}+C_{2} x / \varepsilon+C_{3} y / \varepsilon+O\left(r^{2} / \varepsilon^{2}\right) & \text { if } r<\varepsilon R_{0} \\
e^{2 i \phi}\left(1+O\left(\frac{\varepsilon^{q+1}}{r^{q+1}}\right)\right) & \text { if } r>\varepsilon R_{0}\end{cases} \\
\widetilde{r e^{ \pm 3 i \phi}}= \begin{cases}\varepsilon\left(C_{ \pm 4}+C_{ \pm 5} x / \varepsilon+C_{ \pm 6} y / \varepsilon+O\left(r^{2} / \varepsilon^{2}\right)\right) & \text { if } r<\varepsilon R_{0} \\
r e^{ \pm 3 i \phi}\left(1+O\left(\frac{\varepsilon^{q+1}}{r^{q+1}}\right)\right) & \text { if } r>\varepsilon R_{0}\end{cases}
\end{gathered}
$$

where the $C_{k}$ are constants.

The only smoothing that we have not yet had to estimate is that of the remainder term $r^{2} p(x, y)$. This term will provide a higher order contribution to the metric and therefore will not require a such a delicate estimate in order to be able to differentiate it when we come to calculate the curvature. We may write the smoothing of this term as

$$
\begin{aligned}
\widetilde{r^{2} p(x, y)}=\int_{0}^{\infty} & \int_{0}^{2 \pi}\left(r^{2}+2 \varepsilon \rho \cos (\psi-\phi)+\varepsilon^{2} \rho^{2}\right) \\
& \times p(r \cos \phi+\varepsilon \rho \cos \psi, r \sin \phi+\varepsilon \rho \sin \psi) \\
& \times \Phi(\rho \cos \psi, \rho \sin \psi) \rho d \rho d \psi
\end{aligned}
$$

For $r<\varepsilon R_{0}$, we have

$$
\begin{aligned}
\left.\widehat{r^{2} p(x, y)}\right|_{r=0}= & \varepsilon^{2} \int_{\mathbb{R}^{2}}\left(\lambda^{2}+\mu^{2}\right) p(\varepsilon \lambda, \varepsilon \mu) \Phi(\lambda, \mu) d \lambda d \mu=O\left(\varepsilon^{2}\right), \\
\left.\frac{d}{d x}\left\{\widetilde{r^{2} p(x, y)}\right\}\right|_{r=0}= & 2 \varepsilon \int_{\mathbb{R}^{2}} \lambda p(\varepsilon \lambda, \varepsilon \mu) \Phi(\lambda, \mu) d \lambda d \mu \\
& +\varepsilon^{2} \int_{\mathbb{R}^{2}}\left(\lambda^{2}+\mu^{2}\right) p_{, x}(\varepsilon \lambda, \varepsilon \mu) \Phi(\lambda, \mu) d \lambda d \mu=O(\varepsilon),
\end{aligned}
$$


and similarly

$$
\left.\frac{d}{d y}\left\{\widetilde{r^{2} p(x, y)}\right\}\right|_{r=0}=O(\varepsilon)
$$

Therefore

$$
\widetilde{r^{2} p(x, y)}=M(\varepsilon)+O(\varepsilon r)
$$

where $M(\varepsilon)$ is an $O\left(\varepsilon^{2}\right)$ function of $\varepsilon$ alone.

If however, $r>\varepsilon R_{0}$, then

$$
\begin{aligned}
\widetilde{r^{2} p(x, y)}=r^{2} & \int_{0}^{\infty} \int_{0}^{2 \pi}\left(1+2 \frac{\varepsilon \rho}{r} \cos (\psi-\phi)+\frac{\varepsilon^{2} \rho^{2}}{r^{2}}\right) \\
& \times p(r(\cos \phi+\varepsilon \rho / r \cos \psi), r(\sin \phi+\varepsilon \rho / r \sin \psi)) \\
& \times \Phi(\rho \cos \psi, \rho \sin \psi) \rho d \rho d \psi
\end{aligned}
$$

and so by applying the Mean Value Theorem to $f(s)=p(x+s \lambda, y+s \mu)$

$$
p(x+\lambda, y+\mu)=p(x, y)+\lambda p_{, x}(x+\xi \lambda, y+\xi \mu)+\mu p_{, y}(x+\xi \lambda, y+\xi \mu),
$$

where $\xi \in(0,1)$; it may be concluded that

$$
\widetilde{r^{2} p(x, y)}=r^{2} p(x, y)(1+O(\varepsilon / r))
$$

We now may add up the smoothings in the summation (3) to obtain

$$
\widetilde{h}(x, y)= \begin{cases}C_{1}(\varepsilon)+C_{2}(\varepsilon) \frac{x}{\varepsilon}+C_{3}(\varepsilon) \frac{y}{\varepsilon}+O\left(r^{2} / \varepsilon^{2}\right)+O(r \varepsilon), & \text { if } r<\varepsilon R_{0}, \\ h(x, y)\left(1+O\left(\frac{\varepsilon^{q+1}}{r^{q+1}}\right)\right)+O(r \varepsilon), & \text { if } r>\varepsilon R_{0}\end{cases}
$$

express the smoothed metric $\widetilde{g_{a b}}$ as

$$
\widetilde{g_{a b}}= \begin{cases}C_{a b}^{(0)}(\varepsilon)+C_{a b}^{(1)}(\varepsilon) x / \varepsilon+C_{a b}^{(2)}(\varepsilon) y / \varepsilon+O\left(r^{2} / \varepsilon^{2}\right)+O(r \varepsilon) & \text { if } r<\varepsilon R_{0} \\ g_{a b}+O\left(\frac{\varepsilon^{q+1}}{r^{q+1}}\right)+O(r \varepsilon) & \text { if } r>\varepsilon R_{0}\end{cases}
$$

where $C_{a b}^{(i)}(\varepsilon)$ are $O(1)$ functions of $\varepsilon$ alone, and use it to calculate the Ricci scalar density as

$$
\tilde{R}_{\varepsilon} \sqrt{\tilde{g}_{\varepsilon}}= \begin{cases}O\left(1 / \varepsilon^{2}\right)+O(\varepsilon / r) & \text { if } r<\varepsilon R_{0} \\ O\left(\varepsilon / r^{3}\right) & \text { if } r>\varepsilon R_{0}\end{cases}
$$

To obtain a distributional interpretation to this curvature, we shall follow the method of Clarke et al. (1996). Much of the analysis is applicable, but this time we shall be working with more crude estimates.

Given $\Psi \in \mathcal{D}\left(\mathbb{R}^{2}\right)$, we define

$$
\begin{aligned}
K & =\operatorname{supp} \Psi \\
R_{K} & =\sup \left\{\left(x^{2}+y^{2}\right)^{1 / 2}|| \Psi(x, y) \mid>0\right\} \\
B_{\varepsilon} & =\sup \left\{(x, y) \in \mathbb{R}^{2} \mid\left(x^{2}+y^{2}\right)^{1 / 2}<\varepsilon R_{0}\right\}
\end{aligned}
$$

By the mean value theorem, we may write for some $\xi \in[0,1]$, that

$$
\int_{K} \tilde{R}_{\varepsilon} \sqrt{\tilde{g}_{\varepsilon}}(x, y) \Psi(x, y) d x d y=I_{1}+I_{2}
$$


where

$$
\begin{aligned}
& I_{1}=\int_{K} \tilde{R}_{\varepsilon} \sqrt{\tilde{g}_{\varepsilon}} d x d y \Psi(0,0) \\
& I_{2}=\left.\int_{K} \tilde{R}_{\varepsilon} \sqrt{\tilde{g}_{\varepsilon}} r \frac{d \Psi}{d r}\right|_{(\xi x, \xi y)} d x d y .
\end{aligned}
$$

Now

$$
\begin{aligned}
\left|I_{2}\right| & \leqslant M_{1} \int_{B_{\varepsilon}}\left|\tilde{R}_{\varepsilon} \sqrt{\tilde{g}_{\varepsilon}}\right| r d x d y+M_{1} \int_{K-B_{\varepsilon}}\left|\tilde{R}_{\varepsilon} \sqrt{\tilde{g}_{\varepsilon}}\right| r d x d y \\
& \leqslant 2 \pi M_{2} \frac{\left(\varepsilon R_{0}\right)^{3}}{\varepsilon^{2}}+2 \pi M_{3} \varepsilon^{3} R_{0}^{2}+2 \pi M_{4} \varepsilon\left(\log R_{k}-\log \left(\varepsilon R_{0}\right)\right)
\end{aligned}
$$

where $M_{n}$ are positive constants, giving that $I_{2}=O(\varepsilon \log \varepsilon)$.

To calculate $I_{1}$ we let $D=\left\{(x, y) \mid\left(x^{2}+y^{2}\right)^{1 / 2} \leqslant R_{D}\right\} \subseteq K$. and write

$$
I_{1}=\int_{K-D} \tilde{R}_{\varepsilon} \sqrt{\tilde{g}_{\varepsilon}} d x d y \Psi(0,0)+\int_{D} \tilde{R}_{\varepsilon} \sqrt{\tilde{g}_{\varepsilon}} d x d y \Psi(0,0)
$$

The first integral will be $O\left(\varepsilon / R_{D}\right)$, converging to zero as $\varepsilon \rightarrow 0$. For the second integral the Gauss-Bonnet theorem is applicable. It is also the case that

$$
\int_{\partial D} \kappa_{\tilde{g}_{\varepsilon}} d s=2 \pi A+O\left(\varepsilon / R_{D}\right)
$$

Therefore

$$
I_{1}=4 \pi(1-A) \Psi(0,0)+O\left(\varepsilon / R_{D}\right) .
$$

It may therefore be concluded that

$$
\lim _{\varepsilon \rightarrow 0} \int_{K} \tilde{R}_{\varepsilon} \sqrt{\tilde{g}_{\varepsilon}}(x, y) \Psi(x, y) d x d y=4 \pi(1-A) \Psi(0,0)
$$

giving us

$$
[\tilde{R} \sqrt{-\tilde{g}}] \approx 4 \pi(1-A) \delta^{(2)}(x, y) .
$$

This shows that the distributional curvature associated to the curvature of the generalised metric transforms as a scalar density of weight +1 .

\section{Conclusion}

We have now shown that the calculations of Clarke et al. (1996) are independent under $C^{\infty}$ diffeomorphisms, which suggests that Colombeau's theory could be reformulated in a covariant manner.

There have been many attempts at amending Colombeau's theory in order to achieve coordinate invariance in this sense. One such approach has been to use the simplified algebra (Biagioni, 1990; Colombeau, 1992) in which one defines the space base $\mathcal{E}(\Omega)$ as consisting of functionals dependent on the regularisation parameter $\varepsilon \in(0,1]$, rather than the smoothing kernel $\Phi \in \mathcal{A}_{0}(\Omega)$, thus avoiding the problem of using the spaces $\left.\mathcal{A}_{q} \mathbb{R}^{n}\right)$ in the definitions of $\mathcal{E}_{M}(\Omega)$ and $\mathcal{N}(\Omega)$; however this still does not make the embedding, which involves $\Phi$, commute with $\mu$.

A completely different approach was pursued by Colombeau and Meril (1994) in which they substantially redefined the kernel spaces $\mathcal{A}_{q}\left(\mathbb{R}^{n}\right)$ with weaker moment conditions imposed which does result in the ability to construct $\mathcal{G}(\Omega)$ in a coordinate invariant manner together with the embedding commuting with $\mu$. This formalism enables scalar generalised functions to be constructed, whose transformation laws coincide with those of scalar distributions.

Work is currently in progress (Vickers and Wilson, 1998) into extending the formalism of Colombeau and Meril to enable generalised functions to be constructed as multi-index tensors whose transformation laws coincide with those of tensor distributions. 


\section{Appendix. Smoothing of POLAR FUnCtions}

In this appendix we shall smooth the non-regular complex functions $e^{2 i \phi}$ and $r e^{3 i \phi}$ and hence obtain estimates for the regions $r<\varepsilon R_{0}$ and $r>\varepsilon R_{0}$. For the fine details of calculation the reader is referred to Clarke et al. (1996). We begin with the function

$$
f(x, y)=r^{a-1} e^{i(a+1) \phi}
$$

where $a=1,2$. Its smoothing, with a kernel $\Phi \in \mathcal{A}_{q}\left(\mathbb{R}^{2}\right)$ may be expressed in polar coordinates as

$$
\tilde{f}(\Phi, r \cos \phi, r \sin \phi)=\int \frac{\left(r e^{i \phi}+\varepsilon \rho e^{i \psi}\right)^{a} \Phi(\rho \cos \psi, \rho \sin \psi)}{r^{2}+\varepsilon^{2} \rho^{2}+2 \varepsilon \rho \cos (\psi-\phi)} \rho d \rho
$$

We may expand $\Phi$ as a sum of circular harmonics;

$$
\Phi(r \cos \phi, r \sin \phi)=\sum_{n \in \mathbb{Z}} e^{i n \phi}
$$

so that we may write

$$
\tilde{f}\left(\Phi, r \cos \phi, r \sin \phi=\sum_{n \in \mathbb{Z}} F_{n}(r) e^{i(n+a+1) \phi}\right.
$$

where

$$
F_{n}(r)=r^{a-1} \int \frac{1+\varepsilon \rho / r e^{i \psi}}{1+\varepsilon \rho / r e^{-i \psi}} e^{i n \psi} \Phi_{n}(\rho) \rho d \rho d \psi .
$$

On integrating out $\psi$ we obtain

$$
F_{n}(r)= \begin{cases}2 \pi r^{a-1} \int_{0}^{r / \varepsilon}\left(-\frac{\varepsilon \rho}{r}\right)^{n}\left(1-\frac{\varepsilon^{2} \rho^{2}}{r^{2}}\right)^{a} \Phi_{n}(\rho) \rho d \rho & \text { if } n \geqslant 0 \\
2 \pi r^{a-1} \sum_{k=-n}^{a}\left(\begin{array}{l}
a \\
k
\end{array}\right) \int_{0}^{r / \varepsilon}\left(-\frac{\varepsilon \rho}{r}\right)^{n}\left(-\frac{\varepsilon^{2} \rho^{2}}{r^{2}}\right)^{k} \Phi_{n}(\rho) \rho d \rho & \\
-2 \pi r^{a-1} \sum_{k=0}^{-(n+1)}\left(\begin{array}{l}
a \\
k
\end{array}\right) \int_{r / \varepsilon}^{\infty}\left(-\frac{\varepsilon \rho}{r}\right)^{n}\left(-\frac{\varepsilon^{2} \rho^{2}}{r^{2}}\right)^{k} \Phi_{n}(\rho) \rho d \rho & \text { if }-a \leqslant n \leqslant-1 \\
-2 \pi r^{a-1} \int_{r / \varepsilon}^{\infty}\left(-\frac{\varepsilon \rho}{r}\right)^{n}\left(1-\frac{\varepsilon^{2} \rho^{2}}{r^{2}}\right)^{a} \Phi_{n}(\rho) \rho d \rho & \text { if } n \leqslant-(a+1)\end{cases}
$$

We are interested in estimates for both $r<\varepsilon R_{0}$ and $r>\varepsilon R_{0}$;

To estimate $\tilde{f}$ for $r<\varepsilon R_{0}$ we begin by estimating the integrals

$$
\begin{array}{ll}
\alpha_{n, k}(r)=2 \pi \int_{0}^{r / \varepsilon} \rho^{k+1} \rho^{k+1} \Phi_{n}(\rho) d \rho & k \geqslant 0 \\
\beta_{n, k}(r)=2 \pi \int_{r / \varepsilon}^{\infty} \rho^{k+1} \rho^{k+1} \Phi_{n}(\rho) d \rho & k \leqslant 1
\end{array}
$$

It is easily seen that

$$
\begin{aligned}
& \left|\alpha_{n, k}(r)\right| \leqslant M \frac{r^{k+2}}{\varepsilon^{k+2}} \quad k \geqslant 0 \\
& \left|\beta_{n, k}(r)\right| \leqslant M \frac{r^{k+2}}{\varepsilon^{k+2}} \quad k \leqslant 3
\end{aligned}
$$

The estimates for $\beta_{n, k}$ for $k \geqslant-2$ are more delicate; they must be obtained by Taylor expanding out about $r / \varepsilon=0$;

$$
\begin{aligned}
& \beta_{n,-2}(r)=2 \pi \int_{0}^{\infty} \rho^{-1} \Phi_{n}(\rho) d \rho+O(r / \varepsilon) \\
& \beta_{n,-1}(r)=2 \pi \int_{0}^{\infty} \Phi_{n}(\rho) d \rho+O(r / \varepsilon) \\
& \beta_{n, 0}(r)=2 \pi \int_{0}^{\infty} \rho \Phi_{n}(\rho) d \rho+O\left(r^{2} / \varepsilon^{2}\right) \\
& \beta_{n, 1}(r)=2 \pi \int_{0}^{\infty} \rho^{2} \Phi_{n}(\rho) d \rho+O\left(r^{3} / \varepsilon^{3}\right)
\end{aligned}
$$


We may use the estimates for $\alpha_{n, k}$ and $\beta_{n, k}$ to obtain estimates for $F_{n}$

For $a=1$ we have

$$
F_{n}= \begin{cases}(-1)^{n} \frac{\varepsilon^{n}}{r^{n}}\left(\alpha_{n, n}-\frac{\varepsilon^{2}}{r^{2}} \alpha_{n, n+2}\right) & \text { for } n \geqslant 0 \\ -(-1)^{n} \frac{\varepsilon^{n}}{r^{n}}\left(\beta_{n, n}-\frac{\varepsilon^{2}}{r^{2}} \beta_{n, n+2}\right) & \text { for } n \leqslant-2 \\ \frac{\varepsilon}{r} \alpha_{-1,1}+\frac{r}{\varepsilon} \beta_{-1,-1} & \text { for } n=-1\end{cases}
$$

giving that

$$
F_{n}= \begin{cases}2 \pi \int_{0}^{\infty} \rho \Phi_{-2}(\rho) d \rho+O\left(r^{2} / \varepsilon^{2}\right) & \text { for } n=-2 \\ 2 \pi \frac{r}{\varepsilon} \int_{0}^{\infty} \Phi_{-1}(\rho) d \rho+O\left(r^{2} / \varepsilon^{2}\right) & \text { for } n=-1 \\ -2 \pi \frac{r}{\varepsilon} \int_{0}^{\infty} \Phi_{-3}(\rho) d \rho+O\left(r^{2} / \varepsilon^{2}\right) & \text { for } n=-3 \\ O\left(r^{2} / \varepsilon^{2}\right) & \text { for } n \geqslant 0 \text { or } n \leqslant-4\end{cases}
$$

and for $a=2$ we have

$$
F_{n}= \begin{cases}(-1)^{n} \frac{\varepsilon^{n}}{r^{n-1}}\left(\alpha_{n, n}-2 \frac{\varepsilon^{2}}{r^{2}} \alpha_{n, n+2}+\frac{\varepsilon^{4}}{r^{4}} \alpha_{n, n+4}\right) & \text { for } n \geqslant 0 \\ -(-1)^{n} \frac{\varepsilon^{n}}{r^{n-1}}\left(\beta_{n, n}-2 \frac{\varepsilon^{2}}{r^{2}} \beta_{n, n+2}+\frac{\varepsilon^{4}}{r^{4}} \beta_{n, n+4}\right) & \text { for } n \leqslant-3 \\ \varepsilon\left(2 \alpha_{-1,1}-\frac{\varepsilon^{2}}{r^{2}} \alpha_{-1,3}\right)+\frac{r^{2}}{\varepsilon} \beta_{-1,-1} & \text { for } n=-1 \\ \frac{\varepsilon^{2}}{r} \alpha_{-1,2}-\frac{r^{3}}{\varepsilon^{2}} \beta_{-1,-2}+2 r \beta_{-1,0} & \text { for } n=-2\end{cases}
$$

giving that

$$
F_{n}= \begin{cases}\varepsilon\left(2 \pi \int_{0}^{\infty} \rho^{2} \Phi_{-3}(\rho) d \rho+O\left(r^{2} / \varepsilon^{2}\right)\right) & \text { for } n=-3 \\ \varepsilon\left(2 \pi \frac{r}{\varepsilon} \int_{0}^{\infty} \rho \Phi_{0}(\rho) d \rho+O\left(r^{2} / \varepsilon^{2}\right)\right) & \text { for } n=-2 \\ \varepsilon\left(-2 \pi \frac{r}{\varepsilon} \int_{0}^{\infty} \rho \Phi_{-4}(\rho) d \rho+O\left(r^{2} / \varepsilon^{2}\right)\right) & \text { for } n=-4 \\ \varepsilon O\left(r^{2} / \varepsilon^{2}\right) & \text { for } n \geqslant-1 \text { or } n \leqslant-5\end{cases}
$$

Therefore we have

$$
\begin{aligned}
& \widetilde{e^{2 i \phi}}=2 \pi \int_{0}^{\infty} \rho \Phi_{-2} d \rho+2 \pi \frac{r e^{i \phi}}{\varepsilon} \int_{0}^{\infty} \Phi_{-1}(\rho) d \rho \\
&-2 \pi \frac{r e^{-i \phi}}{\varepsilon} \int_{0}^{\infty} \Phi_{-3}(\rho) d \rho+O\left(r^{2} / \varepsilon^{2}\right) \\
& \widetilde{r e^{3 i \phi}}=2 \pi \varepsilon \int_{0}^{\infty} \rho^{2} \Phi_{-3}(\rho) d \rho+2 \pi r e^{i \phi} \int_{0}^{\infty} \rho \Phi_{0}(\rho) d \rho \\
&-2 \pi r e^{-i \phi} \int_{0}^{\infty} \rho \Phi_{-4}(\rho) d \rho+O\left(r^{2} / \varepsilon\right)
\end{aligned}
$$

On the other hand if $r>\varepsilon R_{0}$ then

$$
F_{n}(r)= \begin{cases}2 \pi r^{a-1} \int_{0}^{\infty}\left(-\frac{\varepsilon \rho}{r}\right)^{n}\left(1-\frac{\varepsilon^{2} \rho^{2}}{r^{2}}\right)^{a} \Phi_{n}(\rho) \rho d \rho, & \text { if } n \geqslant 0, \\
2 \pi r^{a-1} \sum_{k=-n}^{a}\left(\begin{array}{l}
a \\
k
\end{array}\right) \int_{0}^{\infty}\left(-\frac{\varepsilon \rho}{r}\right)^{n}\left(-\frac{\varepsilon^{2} \rho^{2}}{r^{2}}\right)^{k} \Phi_{n}(\rho) \rho d \rho, & \text { if }-a \leqslant n \leqslant-1, \\
0, & \text { otherwise. }\end{cases}
$$

On writing the moment conditions, for $\Phi \in \mathcal{A}_{q}\left(\mathbb{R}^{2}\right)$, in polar coordinates as

$$
\begin{aligned}
& 2 \pi \int_{0}^{\infty} \Phi_{0}(\rho) \rho d \rho=1 \\
& 2 \pi \int_{0}^{\infty} \rho^{c+1} \Phi_{n}(\rho) d \rho=0 \quad 0 \neq|n| \leqslant c \leqslant q, \quad c+n \text { even; }
\end{aligned}
$$


it is easily seen that

$$
\begin{aligned}
& F_{0}(r)=1+O\left(\varepsilon^{q+1} / r^{q+1}\right) \\
& F_{n}(r)=O\left(\varepsilon^{q+1} / r^{q+1}\right), \quad n \neq 0
\end{aligned}
$$

and hence that

$$
\begin{gathered}
\widetilde{e^{2 i \phi}}=e^{2 i \phi}+O\left(\frac{\varepsilon^{q+1}}{r^{q+1}}\right) \\
\widetilde{r e^{3 i \phi}}=r e^{3 i \phi}+O\left(\frac{\varepsilon^{q+1}}{r^{q}}\right)
\end{gathered}
$$

\section{ACKNOWLEDGEMENT}

The authors wish to thank ESI for supporting their visit to the institute as part of the project on Nonlinear Theory of Generalised functions. The authors thank M. Kunzinger, M. Oberguggenberger and R. Steinbauer for helpful discussions. J. Wilson acknowledges the support of EPSRC. grant No. $\mathrm{GR} / 82236$.

\section{REFERENCES}

H. Balasin, Distributional energy-momentum tensor of the extended Kerr geometry, Class. Quantum Grav. 14 (1997), 3353-3362.

H. A. Biagioni, A nonlinear theory of generalised functions, Lecture Notes in Mathematics 1421, Springer, 1990.

C. J. S. Clarke, J. A. Vickers and J. P. Wilson, Generalised functions and distributional curvature of cosmic strings, Class. Quantum Grav. 13, 2485-2498.

J. F. Colombeau, New generalised functions and multiplication of distributions, North-Holland Mathematics Studies 84, North-Holland, 1984

J. F. Colombeau, Multiplication of distributions, Lecture Notes in Mathematics 1532, Springer, 1992.

J. F. Colombeau and A. Meril, Generalised functions and multiplication of distributions on $C^{\infty}$ manifolds, J. Math. Anal. Appl. 186 (1994), 357-364.

R. Steinbauer, The ultrarelativistic Riessner-Nordstrøm field in the Colombeau algebra, J. Math. Phys. 38 (1997), 16141622.

J. A. Vickers and J. P. Wilson, Invariance of the distributional curvature of the cone under smooth diffeomorphisms, Preprint (1998).

Faculty of Mathematical Studies, University of Southampton, Southampton SO17 1BJ, UK.

Email addresses: jav@maths.soton.ac.uk, jpw@maths.soton.ac.uk 